\title{
reviews
}

THE sixth volume in this definitive series devoted to Newton's mathematical papers covers the most important period in his scientific career, the climax of which was the publication of the Principia in 1687. Dr Whiteside has collected mathematically significant extracts from Newton's manuscripts bearing on his researches in the theory of moving bodies and of gravitation, which were his main concern at that time. These comprised the dynamical theory of the motion of bodies under central forces and his attempts on this basis to explain the observed motions of comets and the Moon.

In August 1684, following a challenge from Christopher Wren to produce an explanation of planetary motions, Edmund Halley went to Cambridge to discuss the problem with Newton. That momentous visit revived Newton's interest in the general dynamical theory of moving bodies which had lain dormant since his correspondence with Hooke nearly five years previously on the theory of falling bodies. In his final letter to Newton, Hooke raised the key question of the path of a planet acted on by the attraction of the Sun and, in particular, asked whether an inverse square law of force could make the planet deviate from its instantaneous uniform motion into an elliptical orbit with the Sun at a focus.

Three months after Halley had put the same question to him Newton sent to London the first version of a tract, in Latin, on the motion of bodies in an

\section{Falling bodies}

The Mathematical Papers of Isaac Newton. Volume VI, 1684-1691. Edited by D. T. Whiteside, with assistance of M. A. Hoskin and A. Prag. Pp. xxxiv+ 614. (Cambridge University Press.) $£ 25.00$.

Sorry, for copyright
reasons some images
on this page may not
be available online

rbit. This has been reproduced by Dr Whiteside in the present volume, together with an English translation. It begins with a proof that Kepler's law of areas in generalised form can be deduced for a law of central force. Newton also showed that an elliptical path can be described under laws of force varying either as the distance or as the inverse square of the distance, the centres of force being, respectively, at the centre of the ellipse and at a focus. Among other results, he deduced for the latter case the third law of Kepler. He also went on to establish theorems concerning the motion of a projectile in a medium, resistance being directly proportional to speed.

On seeing this tract, Halley hastened again to see Newton in Cambridge. $\mathrm{He}$ found him already revising and enlarging the text into a full-scale treatise on motion. In the next two years, with Halley's encouragement, the text developed into, at first, two selected books, and ultimately the three that constituted the first edition of the Principia. The texts printed in the present volume illustrate the way in which the first book of the Principia evolved. Dr Whiteside has also included manuscripts concerned with particular problems such as that of the solid of least resistance to motion along its axis in a uniform fluid, the approximate determination of a parabolic cometary path, and the annual advance of the lunar apogee.

The volume concludes with some extracts from a scheme for revising the Principia in the early 1690s that Newton later abandoned.

The present volume maintains the high standards of annotation and printing set by previous volumes.

G. J. Whitrow
THIs book is written by a professional. Dr Wong has plenty of experience in analysing experimental data and he is fully aware that the sophistication of the algebraic treatment must be matched by the quality of the data.

The steady-state derivations of rate equations, which form an introduction, are rigorous and there are helpful tips even for those who have been teaching the subject for some time. Some of the nomenclature is unnecessarily different from the usual and some algebraic shortcuts may make the book difficult for the inexperienced. The author's critical judgement of different steady-state treatments will be very valuable for most of the potential readers. Dalziel coefficients are used with advantage. It is a pity, however, that Dalziel's

\section{Enzyme kinetics}

Kinetics of Enzyme Mechanisms. By J. Tze-Fei Wong. Pp. xii +294. (Academic: London and New York, August 1975.) $£ 7.80 ; \$ 20.25$.

treatment is not also used as an extended example for three substrate reactions. The warning about the caution necessary when playing pingpong with Cleland (p.113) should be found in more treatments of enzyme kinetics.

Although it is good to see that the author has not completely neglected transient and relaxation kinetics, this area is not treated satisfactorily. Detail is given of the lag phase treatment developed by the reviewer 20 years ago. This has turned out to be of much less practical application than the subsequent analysis of the transient formation of enzyme bound products. The considerable amount of useful information obtained from this latter use of rapid reaction techniques deserves more space. Similarly the examples given for relaxation studies are those found in other text books. More recent and varied references would have been appreciated.

The above criticisms are obviously very personal ones and should not be taken too seriously. I would certainly recommend to any graduate student and research worker, who has some basic understanding of kinetics and calculus, to use this book for at least part of his journey towards a professional training in enzymology.

H. Gutfreund 